$n=2$, states of the transition, low pressure hydrogen gas must be subject to a gentle electrical discharge as it is pumped through the absorption cell. But electric and magnetic fields present during the discharge would broaden the transitions of interest. Optical observation is therefore delayed by electronic means until 1 microsecond after the discharge is switched off. Furthermore, two devices discriminate in favour of the atomic absorption and against noise signals. First, the probe beam is compared with a similar beam from the same laser which also traverses the gas but along a different, parallel path. The effects of random laser amplitude fluctuations are thereby reduced. Second, the saturating beam is amplitude modulated by a simple chopping method while the probe beam is amplified at the chopping frequency by a narrow band amplifier. This ensures that only absorptions due to atoms simultaneously subject to both beams are detected, because only then will the probe be modulated at the chopping frequency.

Advantages other than the further confirmation of important theoretical predictions should accrue from refined versions of this experiment. For example, as it is pointed out, a value of the Rydberg constant of accuracy comparable with the present standard of length should be obtainable. Because the Rydberg constant depends only on a few fundamental constants (one of which is Planck's constant), such an improved value would help one to a better knowledge of these important quantities.-.. A. C. L.

\section{Chemotactic Signals}

EMBRYONIC development is characterized by the organized growth, differentiation and movement of fields of cells. It is suspected that such global phenomena are controlled by embryonic systems of communication which may be analogous to the functional control systems in adult organisms. The nature of such communication remains an obscure and fascinating problem.

In last week's Nature New Biology $(\mathbf{2 3 5}, 90 ; 1972)$ and in a previous publication (Biochem. Biophys. Res. Commun., 42, 119 ; 1971), Gerisch et al. report an investigation of a morphogenetic movement which is currently probably the best understood example of a field phencmenon-the aggregative movement of the cellular slime mould Dictyostelium discoideum in which freeliving slime mould cells are induced on starvation to begin their development by aggregating chemotactically. Each aggregate ultimately forms a fruiting body (adult stage). The aggregation occurs by a movement response to periodic signals, which are propagated centrifugally through each aggregation field. It is almost certain that the signals are pulses of $3^{\prime}-5^{\prime}$-cyclic AMP (cyclic AMP) and that they are propagated by a relaying system, whereby a cell which receives a signal responds by making one. The cells secrete an extracellular cyclic AMP phospho. diesterase (PD), which is traditionally considered to reduce noise in the system.

Gerisch et al. are concerned with regulation of the PD activity, and report that whereas suspension cultures of vegetative $D$. discoideum cells have a high extracellular level of PD activity, the activity drops in starved cell sus- pensions. They have shown that this is caused by the appearance of an extracellular PD inhibitor which is a heat stable protein (molecular weight 40,000 ) and is specific in the sense of not inhibiting beef heart PD or another extracellular $D$. discoideum enzyme ( $\mathrm{N}$-acetyl glucosaminidase). The inhibitor appears one to two hours after starvation (aggregation occurs after eight hours) and causes a rapid fifty-fold drop in activity of the enzyme, which is followed by a slow rise (to ten-fold after eight hours).

Gerisch et al. examined twenty-three morphogenetic mutants of $D$. discoideum which failed either to aggregate or had unusual aggregation and six species of slime moulds related to $D$. discoideum for their PD-inhibitor systems. Twelve of the twenty-three mutants had defects in PD control (ranging from absence of PD or inhibitor to timing defects). In the three cases where the relationship between morphogenetic defects and PD-inhibitor defects is given, one mutant which failed to aggregate had no PD inhibitor and two mutants with giant aggregations had very low PD activity. Among the six species, three, including $D$. discoideum, which co-aggregate and presumably have similar movement control systems, had similar and cross-reacting PD-inhibitor systems whereas three which fail to co-aggregate with $D$. discoideum had either very little extracellular PD and no PD inhibitor (two cases) or an extracellular PD activity but no heatstable inhibitor (one case).

These findings suggest that the PD has a vital role in the aggregation of $D$. discoideum. Gerisch et al. suggest that its primary role may not be to dissipate signal noise, but rather to control differentiation of aggregation competence by way of regulation of the external level of cyclic AMP. This function would be consistent with the time course of PD activity and with the observation that extracellular cyclic AMP $\left(10^{-3} \mathbf{M}\right)$ causes changes in $D$. dis. coideum cells which are associated with the aggregative and later stages of development (for example, it promotes cellular adhesion). Gerisch et al. report two more findings to support their contention: namely, that a cold shock at the end of the growth phase suppresses both inhibitor production and the onset of aggregation competence of cell suspensions of $D$. discoideum and that beef heart PD, which is not affected by the $D$. discoideum inhibitor, retards the onset of aggregation competence in the slime mould.

One criticism of this study is that the experiments have been performed on cells in liquid culture, whereas $D$. discoideum normally aggregates only at an air-solid interface. Starved liquid culture cells undergo changes observed in aggregating cells and will aggregate after a reduced time if deposited on a solid surface, but the precise relation of this differentiation to differentiation in normal development remains in some doubt. Unfortunately, no quantitative measurements are available for PD in aggregating cells.

This investigation provides a glimpse of the insight into functioning of developmental control systems which may result from attempting to relate molecular to morphogenetic parameters in a suitable experimental system. It is clear that full understanding of the $D$. discoideum signalling system and of the role of the PD in its functioning will depend on measurement of the other signal and response parameters and investigation of their interactions, but Gerisch et al.'s investigations are an exciting step in the right direction.-From a correspondent. 